\title{
Estimativas DA EVAPOTRANSPIRAÇÃo DE REFERÊNCIA PARA O MUNICÍPIO DE Apodi, RN
}

\author{
Leandro Moscôso Araújo ${ }^{1}$, Francisco Thiago Coelho Bezerra², Péricles de Farias Borges ${ }^{3}$, Alécio Rodrigues \\ Pereira ${ }^{4}$, Janielly Silva Costa Moscôso ${ }^{5}$, Lázaro de Souto Araújo ${ }^{3}$
}

\author{
${ }^{1}$ Universidade Estadual Paulista - UNESP, Faculdade de Ciências Agronômicas, Programa de Pós-Graduação em Agronomia/Irrigação e Drenagem, \\ Botucatu, SP. \\ ${ }^{2}$ Universidade Federal da Paraíba, Centro de Ciências Agrárias, Programa de Pós-Graduação em Agronomia. Areia, PB. \\ ${ }^{3}$ Universidade Federal da Paraíba, Centro de Ciências Agrárias, Departamento de Ciências Fundamentais e Sociais. Areia, PB. \\ ${ }^{4}$ Universidade Federal de Campina Grande, Centro de Tecnologia e Recursos Naturais, Programa de Pós-Graduação em Meteorologia, Campina Grande, PB. \\ ${ }^{5}$ Universidade Federal de Santa Maria, Centro de Ciências Rurais, Programa de Pós-Graduação em Ciência do Solo, Santa Maria, RS. \\ * Autor para correspondência: periclesufpb@gmail.com
}

Recebido em 22 de junho de 2017. Aceito em 21 de dezembro de 2018. Publicado em 28 de dezembro de 2018.

Resumo - A estimativa da evapotranspiração é um relevante componente no manejo da irrigação e, sua mensuração envolve diversos parâmetros que podem dificultar na sua obtenção. O trabalho objetivou comparar modelos matemáticos para determinação da evapotranspiração de referência para o município de Apodi, RN. Foram utilizados dados atmosféricos dos meses de setembro à dezembro de 2008 e 2009, fornecidos pelo Instituto Nacional de Meteorologia (INMET). A evapotranspiração de referência foi estimada pelos modelos de Penman-Monteith (Padrão da FAO), Jensen-Haise, HargreavesSamani e Makkink. Foi realizada análise de regressão linear entre os métodos alternativos e o método de Penman-Monteith com os dados de 2008. A partir dos modelos de regressão, os dados foram ajustados pelo coeficiente de regressão para os anos de 2008 e 2009. A partir desses dados simulados, calculou-se os índices de Willmott (d), de Pearson (e), de confiabilidade e, a percentagem do desvio (PD). Resultados mais precisos foram encontrados em modelos que utilizam a radiação solar como variável. Os melhores desempenhos foram encontrados para os métodos de Jensen-Haise e Makkink, obtendo correlações classificados como "muito alta". Contudo, os melhores métodos a serem aplicados para a região de Apodi-RN, entre os meses de setembro e dezembro, são o de Jensen-Haise ajustado pelo fator de 0,65 e o de Makkink.

Palavras-chave: Penman-Monteith; coeficiente de Pearson; Jensen-Haise.

\section{REFERENCE EVAPOTRANSPIRATION ESTIMATES FOR the APODI, RN MUNiCIPALITY}

ABSTRACT - The estimate evapotranspiration is an important component in irrigation management, and their measurement involves many parameters that may hamper their achievement. The objective of this work was to compare mathematical models to determine the reference evapotranspiration for the municipality of Apodi, RN. Atmospheric data were used from September to December 2008 and 2009, provided by the National Institute of Meteorology (INMET). The reference evapotranspiration was estimated by the Penman-Monteith (FAO Standard), Jensen-Haise, Hargreaves-Samani and Makkink models. Linear regression analysis was performed between the alternative methods and the Penman-Monteith method with data from 2008. From the regression models, the data were adjusted by regression coefficient for the years 2008 and 2009. From these simulated data, the Willmott (d), Pearson (e), reliability, and the percentage of deviation (PD) were calculated. More accurate results were found in models that use solar radiation as a variable. The best performances were found for the methods of Jensen-Haise and Makkink, obtaining correlations classified as "very high." However, the best methods to be applied to the Apodi-RN region between the months of September and December are the Jensen-Haise adjusted by a factor of 0.65 and Makkink.

Keywords: Penman-Monteith; Pearson's coefficient; Jensen-Haise. 


\section{ESTIMACIONES DE EVAPOTRANSPIRACIÓN DE REFRENCIA PARA EL MUNICIPIO DE APODI, RN}

Resumen - Estimación de la evapotranspiración es un componente importante en la gestión del riego y su medición implica muchos parámetros que pueden dar lugar a su logro. El objetivo del estudio fue comparar los modelos matemáticos para determinar la evapotranspiración de referencia para la ciudad de Apodi, RN. Se utilizaron datos atmosféricos de los meses de septiembre a diciembre de 2008 y 2009, proporcionados por el Instituto Nacional de Meteorología (INMET). La evapotranspiración de referencia fue estimada por los modelos de Penman-Monteith (Estándar de la FAO), Jensen-Haise, Hargreaves-Samani y Makkink. Se realizó un análisis de regresión lineal entre los métodos alternativos y el método de Penman-Monteith con los datos de 2008. A partir de los modelos de regresión, los datos fueron ajustados por el coeficiente de regresión para los años 2008 y 2009. A partir de estos datos simulados, se calcularon los índices Willmott (d), Pearson (e), la fiabilidad y la desviación porcentual (PD). Se encontraron resultados más precisos en los modelos que utilizan la radiación solar como una variable. Se han encontrado los mejores rendimientos para los métodos de Jensen-Haise y Makkink, logrando correlaciones clasificados como "muy alta". Sin embargo, los mejores métodos aplicarse en la región Apodi-RN, entre los meses de septiembre y diciembre, son los Jensen-Haise ajustado por un factor de 0.65 y el de Makkink.

Palabras clave: Penman-Monteith; coeficiente de Pearson; Jensen-Haise.

\section{INTRODUÇÃO}

Em regiões áridas e semiáridas, a precipitação pluviométrica é caracterizada como baixa, irregular e distribuída em poucos meses. Esse fator meteorológico torna-se, em parte, um entrave para a agricultura de sequeiro dessas áreas. Porém, facilita o planejamento de uma agricultura irrigada, desde que haja água de qualidade e em quantidade suficiente.

$\mathrm{Na}$ agricultura irrigada, o manejo da irrigação é um fator decisivo para o sucesso do empreendimento. A estimativa da evapotranspiração de referência $\left(\mathrm{ET}_{0}\right)$ para tal é um parâmetro crucial para no balanço hídrico, estabelecendo um desenvolvimento sustentável que se aplique a gestão de águas (Bezerra et al., 2014), reduzindo perdas e maximizando o rendimento das culturas.

Os modelos utilizados para determinar a $\mathrm{ET}_{0}$ fazem uso de parâmetros meteorológicos e da cultura. Conforme Jensen et al. (1990), a utilização de um modelo em uma determinada região subtende-se que só é válido para um local específico, sendo muito importante realizar a calibração para outras regiões onde se deseja utiliza-lo, levando em consideração as características edafoclimáticas do local.

Para se estimar a evapotranspiração de referência, deve-se levar em consideração alguns fatores limitantes para a sua determinação, tais como: a temperatura do ar (determina a quantidade de vapor que é responsável pela evaporação na superfície das plantas), a umidade relativa do ar (determina a intensidade do fluxo de vapor que irá causar a evaporação e transpiração nas superfícies), a velocidade do vento (proporciona a remoção do ar úmido que está diretamente em contato com a superfície de evaporação ou transpiração) e a radiação solar (a partir do processo de fluxo de calor latente, vai promover a evaporação da superfície dependendo apenas da quantidade de energia disponível).

O método padrão para o cálculo da $\mathrm{ET}_{0}$ é de Penman-Monteith (1948), não somente porque tem base física sólida, mas por apresentar parametrização proposta pela FAO (Allen et al., 1998) e por utilizar um número considerável de variáveis, tornando-se o mais preciso.

Devido aos inúmeros parâmetros utilizados, o método de Penman-Monteith requer a utilização de conjunto de equipamentos, elevando os custos. Dessa forma, outros métodos menos complexos foram desenvolvidos para a estimativa da evapotranspiração de referência, tais como Makkink (1957), Jensen-Haise (1963), Hargreaves-Samani (1985), entre outros. Porém, os resultados desses métodos devem ser correlacionados ao método de PenmanMonteith, garantindo precisão na estimativa da evapotranspiração de referência. 
Diante do exposto, o presente trabalho teve como objetivos determinar o método alternativo e um fator de aproximação para estimar a evapotranspiração de referência.

\section{Material e Métodos}

\section{Caracteristicas da área experimental}

As campanhas experimentais foram realizadas na Chapada do Apodi, oeste do estado do Rio Grande do Norte, localizada no município de Apodi (5³7'37’'S; 3749'54”'W; $138 \mathrm{~m}$ de altitude).

O clima da região, de acordo com Thornthwaite (1948), é semiárido do tipo DA'da', com precipitação média anual de $920 \mathrm{~mm}$, concentrada no verão e outono. As temperaturas média, máxima e mínima são $28,35^{\circ} \mathrm{C}, 33,8^{\circ} \mathrm{C}$ e $22,9^{\circ} \mathrm{C}$, respectivamente. A textura do solo da área experimental é Franco-Argilo-Arenosa, com 56,8\% de areia, $33,7 \%$ de argila e $9,5 \%$ de silte.

\section{Aquisição dos dados}

As informações meteorológicas necessárias para a realização do estudo foram fornecidas pelo Instituto Nacional de Meteorologia (INMET), referentes aos meses de setembro a dezembro de 2008 e de 2009. A Estação Automática responsável pela captação dos dados, está localizada a uma altitude de 150 metros a $-5,6272^{\circ}$ de latitude sul e a $-37,8319^{\circ}$ de longitude oeste, Apodi-RN.

As variáveis utilizadas para a determinação da evapotranspiração de referência foram: temperatura do ar máxima (Tmáx) e mínima (Tmin), umidade relativa do ar máxima (URmáx) e mínima (URmin), radiação solar (Rs) e, velocidade do vento $(\mathrm{U})$ a dois metros de altura (Tabela 1).

Os valores da evapotranspiração de referência diários foram calculados através do software SEVAP (Sistema de Estimativa da Evapotranspiração).

Tabela 1. Dados médios de temperatura do ar, umidade relativa do ar, radiação solar global e velocidade do vento para os meses de setembro, outubro, novembro e dezembro dos anos de 2008 e $2009 \mathrm{em}$ Apodi, $R N$. $T_{\text {máx }}=$

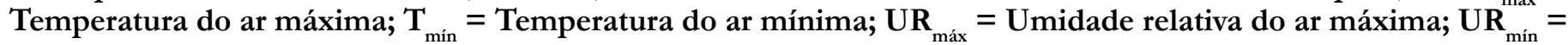
Umidade relativa do ar mínima; $R_{s}=$ Radiação solar global; $U_{2}=$ Velocidade do vento a $2 \mathrm{~m}$

\begin{tabular}{|c|c|c|c|c|c|c|}
\hline 2008 & $\mathrm{~T}_{\text {máx }}$ & $\mathrm{T}_{\min }$ & $\mathrm{UR}_{\text {máx }}$ & $\mathrm{UR}_{\min }$ & $\mathrm{R}_{\mathrm{s}}$ & $\mathrm{U}_{2}$ \\
\hline Mês & \multicolumn{2}{|c|}{$\mathrm{C}^{\mathrm{o}}$} & \multicolumn{2}{|c|}{$\%$} & $\mathrm{MJ} \mathrm{m} \mathrm{m}^{-2} \mathrm{dia}^{-1}$ & $\mathrm{~m} \mathrm{~s}^{-1}$ \\
\hline SET & 28,9 & 27,2 & 58 & 52 & 34,92 & 2,7 \\
\hline OUT & 29,6 & 28,1 & 55 & 49 & 37,87 & 2,9 \\
\hline $\mathrm{NOV}$ & 29,6 & 28,0 & 57 & 51 & 37,55 & 2,9 \\
\hline DEZ & 29,6 & 28,2 & 59 & 53 & 31,56 & 2,7 \\
\hline 2009 & $\mathrm{~T}_{\text {máx }}$ & $\mathrm{T}_{\min }$ & $\mathrm{UR}_{\text {máx }}$ & $\mathrm{UR}_{\text {min }}$ & $\mathrm{R}_{\mathrm{s}}$ & $\mathrm{U}_{2}$ \\
\hline Mês & \multicolumn{2}{|c|}{$\mathrm{C}^{\mathrm{o}}$} & \multicolumn{2}{|c|}{$\%$} & $\mathrm{MJ} \mathrm{m} \mathrm{m}^{-2} \mathrm{dia}^{-1}$ & $\mathrm{~m} \mathrm{~s}^{-1}$ \\
\hline SET & 27,6 & 26,1 & 68 & 61 & 30,53 & 2,2 \\
\hline OUT & 28,4 & 26,8 & 64 & 58 & 33,02 & 2,6 \\
\hline $\mathrm{NOV}$ & 29,2 & 27,6 & 60 & 54 & 31,63 & 2,8 \\
\hline DEZ & 29,1 & 27,6 & 65 & 59 & 27,96 & 2,5 \\
\hline
\end{tabular}


Equação de Penman-Monteith-FAO (EToPM)

A evapotranspiração de referência diária foi obtida através do método da FAO (Allen et al. (1998), que trata de uma cultura hipotética com altura de $12 \mathrm{~cm}$, resistência aerodinâmica da superfície $70 \mathrm{~s} \mathrm{~m}^{-1}$ e albedo de $23 \%$.

$$
\mathrm{ET}_{0} \mathrm{PM}=\frac{0,408 \Delta\left(\mathrm{R}_{\mathrm{n}}-\mathrm{G}\right)+\gamma\left(\frac{900}{\mathrm{~T}_{\text {med }}+273}\right) \mathrm{U}_{2 \mathrm{~m}}\left(\mathrm{e}_{\mathrm{s}}-\mathrm{e}_{\mathrm{a}}\right)}{\Delta+\gamma\left(1+0,34 \mathrm{U}_{2 \mathrm{~m}}\right)}
$$

Em que:

$\mathrm{ET}_{0} \mathrm{PM}=$ evapotranspiração de referência diária da cultura $\left(\mathrm{mm} \mathrm{dia}^{-1}\right)$;

$\mathrm{R}_{\mathrm{n}}=$ saldo de radiação à superfície $\left(\mathrm{MJ} \mathrm{m}^{-2}\right)$;

$\mathrm{G}=$ fluxo de calor no solo $\left(\mathrm{MJ} \mathrm{m}^{-2}\right)$;

$\mathrm{T}_{\text {med }}=$ temperatura média do ar a 2 metros de altura $\left({ }^{\circ} \mathrm{C}\right)$;

$\mathrm{U}_{2 \mathrm{~m}}=$ velocidade média diária do vento a 2 metros de altura da superfície do solo $\left(\mathrm{m} \mathrm{s}^{-1}\right)$;

$\mathrm{e}_{\mathrm{a}}=$ pressão atual (ou parcial) do vapor d'água $(\mathrm{kPa})$;

$\mathrm{e}_{\mathrm{s}}=$ pressão média de saturação do vapor d'água $(\mathrm{kPa})$;

$\Delta=$ inclinação da curva de pressão de saturação de vapor d'água $\left(\mathrm{kPa}^{\circ} \mathrm{C}^{-1}\right) \mathrm{e}$;

$\gamma=$ fator psicrométrico $\left(\mathrm{kPa}^{\circ} \mathrm{C}^{-1}\right)$.

\section{Equação de Jensen-Haise}

Em trabalhos realizados nas regiões semiáridas americanas, Jensen-Haise (1963), propuseram a seguinte relação para $\mathrm{ET}_{0}\left(\mathrm{~mm} \mathrm{dia}^{-1}\right)$ (Pereira et al., 1997):

$$
\mathrm{ET}_{0} \mathrm{JH}=\mathrm{Rs} .(0,025 \mathrm{~T}+0,078)
$$

Em que:

$\mathrm{ET}_{0} \mathrm{JH}=$ evapotranspiração de referência $\left(\mathrm{mm} \mathrm{dia}^{-1}\right)$;

$\mathrm{Rs}=$ radiação solar ao nível do solo $\left(\mathrm{MJ} \mathrm{m}^{-2}\right) \mathrm{e}$;

$\mathrm{T}=$ temperatura média do período $\left({ }^{\circ} \mathrm{C}\right)$.

\section{Equação de Hargreaves-Samani}

O método proposto por Hargreaves e Samani (1985) é uma alternativa para situações em que não se têm dados medidos de radiação solar à superfície, umidade relativa do ar e velocidade do vento:

$$
\mathrm{ET}_{0} \mathrm{HS}=0,0023 \mathrm{Ro}\left(\mathrm{T}_{\text {max }}-\mathrm{T}_{\text {min }}\right)^{0,5} .(\mathrm{T}+17,8)
$$

Em que:

$\mathrm{ET}_{0} \mathrm{HS}=$ evapotranspiração de referência $\left(\mathrm{mm} \mathrm{dia}^{-1}\right)$;

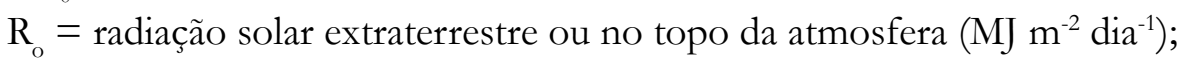

$\mathrm{T}_{\text {máx }}=$ temperatura máxima diária $\left({ }^{\circ} \mathrm{C}\right) \mathrm{e}$;

$\mathrm{T}_{\text {mín }}=$ temperatura mínima diária $\left({ }^{\circ} \mathrm{C}\right)$. 
Makkink (1957) obteve uma correlação entre ETp diária $\left(\mathrm{mm} \mathrm{dia}^{-1}\right)$ e a radiação solar ao nível da superfície expressa em equivalente de evaporação (Rs, $\mathrm{mm} \mathrm{dia}^{-1}$ ) usando dados de evapotranspiração potencial de um gramado em lisímetro de lençol freático constante (Pereira et al., 1997):

$$
\mathrm{ET}_{0} \mathrm{M}=0,61 \text {.W.Rs-0,12 }
$$

Em que:

$\mathrm{ET}_{0} \mathrm{M}=$ evapotranspiração de referência $\left(\mathrm{mm} \mathrm{dia}^{-1}\right)$;

$\mathrm{R}_{\mathrm{s}}=$ radiação solar global $\left(\mathrm{MJ} \mathrm{m}^{-2}\right) \mathrm{e}$;

$\mathrm{W}$ = fator de ponderação que dependente da temperatura do bulbo molhado $(\mathrm{Tu})$.

\section{Análise dos dados}

Os dados foram submetidos a análise de regressão linear entre os métodos alternativos (Jensen-Haise, Hargreaves-Samani e Makkink) e de Penman-Monteith, utilizando as estimativas da evapotranspiração de referência

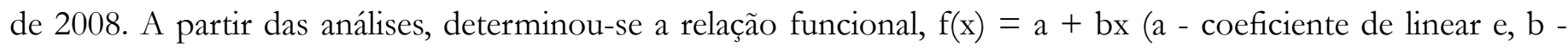
coeficiente angular), entre o método alternativo e o método padrão. A significância do coeficiente regressão foi determinado pelo teste $\mathrm{t}$ de Student $(\mathrm{p} \leq 0,05)$.

Baseado na relação funcional, estimou-se a evapotranspiração de referência de Penaman-Monteith em função do método alternativo.

Para se determinar o método alternativo de maior ajuste ao método de Penaman-Monteith utilizou os parâmetros: coeficientes de determinação $\left(\mathrm{R}^{2}\right)$; índice de Willmott (d) (Willmott, 1981); índice de Pearson (@); índice de confiabilidade, $\mathrm{c}=\mathrm{d}^{*} \varrho$ (Camargo e Sentelhas, 1997) e; percentagem do desvio (PD).

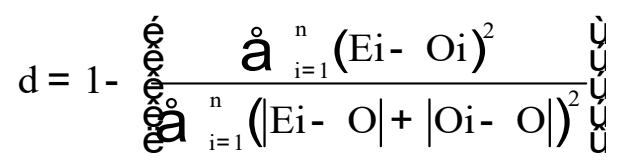

Em que:

$\mathrm{d}=$ índice de Willmott;

$\mathrm{Ei}=$ valores estimados na observação $i$;

Oi = variável observada na observação i e;

$\mathrm{O}=$ média dos valores observados no período;

$$
r=\frac{a_{i=1}^{n}(x i-\bar{x})(y i-\bar{y})}{\sqrt{\stackrel{\circ}{i=1}_{i=1}^{n}(x i-\bar{x})^{2}} \sqrt{\grave{a}_{i=1}^{n}(y i-\bar{y})^{2}}}
$$

Em que:

$\varrho=$ índice de Pearson;

xi $=$ valores observados na observação i;

$\overline{\mathrm{x}}=$ valor médio dos dados observados no período;

yi = valores estimados na observação i e;

$\bar{y}=$ valor médio dos dados estimados no período.

$$
P D=\sqrt{\frac{\stackrel{\circ}{a}_{i=1}^{n}(E i-O i)^{2}}{n}}+\frac{100}{x}
$$


Em que:

$\mathrm{PD}=$ percentagem de desvio;

$\mathrm{Ei}=$ valores estimados na observação i;

Oi = variável observada na observação i;

$\mathrm{n}=$ tamanho da amostra e;

$\mathrm{x}=$ valor médio dos dados observados no período;

A confiabilidade das equações foi testada através do índice de confiança “c” proposta por Camargo e Sentelhas (1997) descrito da seguinte forma:

$$
c=d^{*} r
$$
Em que:
$\mathrm{c}=$ índice de desempenho;
$\mathrm{d}=$ índice de Willmott e;
$\varrho=$ índice de Pearson.

A Tabela 2 apresenta critério adotado para classificar os métodos através do índice "c":

Tabela 2. Critério para classificação dos métodos de estimativa da $\mathbf{E T}_{0}$ (Evapotranspiração de Referência) proposto por Camargo e Sentelhas (1997).

\begin{tabular}{cc}
\hline Índice "c" & Desempenho \\
\hline$>0,85$ & Ótimo \\
0,76 a 0,85 & Muito Bom \\
0,66 a 0,75 & Bom \\
0,61 a 0,65 & Mediano \\
0,51 a 0,60 & Sofrível \\
0,41 a 0,50 & Ruim \\
$\leq 0,40$ & Péssimo \\
\hline
\end{tabular}

Para as análises dos dados utilizou-se o software para análises estatísticas, BioEstat versão 5.0 (Ayres et al., 2007).

\section{Resultados e Discussão}

As regressões lineares entre os valores da evapotranspiração de referência calculados pelos métodos de JansenHaise $\left(\mathrm{ET}_{0} \mathrm{JH}\right)$, Hargreaves-Samani (ET $\left.\mathrm{E}_{0} \mathrm{HS}\right)$ e Makkink (ET $\mathrm{E}_{0}$ ) com o de Penman-Monteith (ET $\mathrm{PM}$ ) geraram modelos com parâmetros ( $a$ e $b$ ) e coeficiente de determinação $\left(\mathrm{R}^{2}\right)$ distintos. Entre os métodos de $\mathrm{ET}_{0} \mathrm{JH}$ e $\mathrm{ET}_{0} \mathrm{PM}$, gerou-se um modelo com boa explicação da variação total, que foi de $72 \%$ (Figura 1A). Enquanto que para a regressão linear entre os valores de evapotranspirações obtidas pelos métodos de $\mathrm{ET}_{0} \mathrm{HS}_{\mathrm{S}}$ e de ET $\mathrm{T}_{0} \mathrm{PM}$, não se obteve um modelo confiável, com coeficiente de determinação de 38\% (Figura 1B). Já entre os métodos de $\mathrm{ET}_{0} \mathrm{M}$ e de ET $\mathrm{PM}_{0}$, obteve-se um coeficiente de determinação de 67\% considerado confiável (Figura 1C). 
Figura 1. Análise de regressão linear entre a evapotranspiração de referência calculada pelos métodos de JasenHaise e Penman-Monteith (A), Hargreaves-Samani e Penman-Monteith (B) e, Makkink e Penman-Monteith (C) a partir dos dados meteorológicos dos meses de setembro a dezembro de 2008, Apodi-RN. **: significativo a 1\% de probabilidade pelo teste $t$.
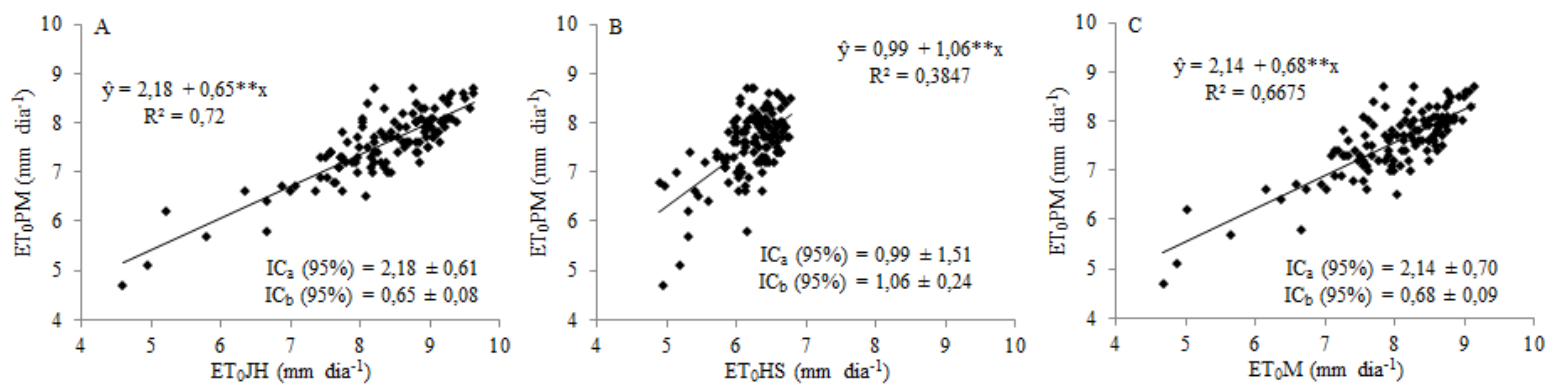

Em estudos relacionados aos métodos de estimativa da evapotranspiração de referência para o chapadão de sul, no Estado do Mato Grosso do Sul, Cunha et al. (2013) encontraram resultados similares aos apresentados neste estudo, no qual os métodos de $\mathrm{ET}_{0} \mathrm{JH}, \mathrm{ET}_{0} \mathrm{HS}$ e $\mathrm{ET}_{0} \mathrm{M}$ correlacionados com o método de $\mathrm{ET}_{0} \mathrm{PM}$ apresentaram valores de 76, 57 e 70\%, respectivamente, no período de janeiro de 2008 a dezembro de 2011.

Após o ajuste da regressão linear entre métodos de cálculo da ETo, a equação gerada foi utilizada para simular os valores a serem obtidos pelo método de $\mathrm{ET}_{0} \mathrm{PM}$ por cada método alternativo.

Como o método de ET $\mathrm{JH}_{0}$ superestima os valores de ETo, tomando como referência o método de ET $\mathrm{PM}_{0}$ em torno de 10,2\%, o valor calculado por este método foi corrigido pelo fator angular da reta de 0,65 (Figura 1A). $\mathrm{Na}$ Figura 2, podem ser observados os valores da ETo calculados pelos métodos de ET $\mathrm{PM}_{0}$ e de ET 0 H, como também os valores simulados pelo ajuste da regressão linear (ET PM - ET 0 H). No ano de 2008, entre os meses de setembro à dezembro, a evapotranspiração médias foi de 7,56 \pm 0,69 calculado pelo método de ET $\mathrm{PM}_{0}$, 8,33 \pm 0,90 calculado pelo método de ET $\mathrm{JH}_{0}$ e de 7,59 \pm 0,59 calculado pelo método de ET $\mathrm{JH}_{0} \mathrm{H}$ corrigida para ET $\mathrm{PM}_{0}$ Em 2009, obteve-se para este mesmo período, as seguintes médias 6,99 \pm 0,85, 7,96 \pm 1,13 e, 7,35 \pm 0,74 quando estimados pelos métodos de $\mathrm{ET}_{0} \mathrm{PM}, \mathrm{ET}_{0} \mathrm{JH}$ e $\mathrm{ET}_{0} \mathrm{JH}$ corrigido para $\mathrm{ET}_{0} \mathrm{PM}$, respectivamente.

Figura 2. Evapotranspiração de referência calculado pelos métodos de Penman-Monteith (-), Penman-Jensen (----) e Jensen-Haise (…...) nos anos de 2008 (A) e 2009 (B), Apodi-RN.
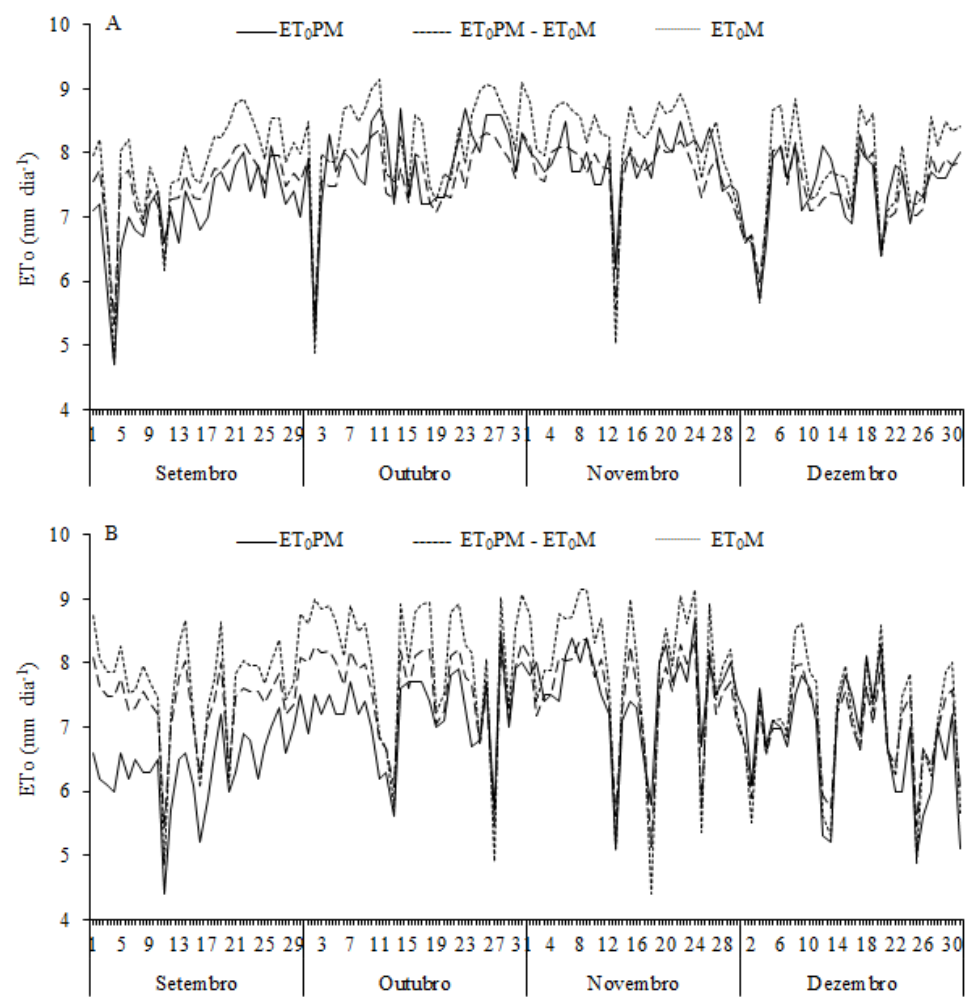
Os dados de evapotranspiração calculados pelo método de $\mathrm{ET}_{0} \mathrm{JH}$ e ajustados para o método de $\mathrm{ET}_{0} \mathrm{PM}$, para os anos de 2008 (Figura 2A) e de 2009 (Figura 2B), foram aproximados satisfatoriamente, como demonstram os indicadores estatístico contidos na Tabela 3. Como o índice de concordância de Willmott (d) acima de 0,8, correlação de Pearson (e) acima de 0,8, o índice de desempenho de Camargo (c) acima de 0,7 e, baixo valor da percentagem de desvio (PD).

Tabela 3. Indicadores estatísticos do desempenho dos modelos.

\begin{tabular}{|c|c|c|c|}
\hline \multirow{2}{*}{ Indicadores } & $\mathrm{ET}_{0} \mathrm{JH}$ & $\mathrm{ET}_{0} \mathrm{HS}$ & $\mathrm{ET}_{0} \mathrm{MK}$ \\
\hline & \multicolumn{3}{|c|}{2008} \\
\hline Willmott (d) & 0,91 & 0,71 & 0,89 \\
\hline Pearson (e) & 0,85 & 0,62 & 0,82 \\
\hline Confiabilidade (c) & 0,78 & 0,44 & 0,73 \\
\hline Percentagem de desvio (PD) & 4,77 & 7,10 & 5,22 \\
\hline \multicolumn{4}{|l|}{ Classificação } \\
\hline & \multicolumn{3}{|c|}{2009} \\
\hline Willmott (d) & 0,88 & 0,63 & 0,84 \\
\hline Pearson $(\varrho)$ & 0,84 & 0,62 & 0,76 \\
\hline Confiabilidade (c) & 0,74 & 0,39 & 0,64 \\
\hline Percentagem de desvio (PD) & 8,37 & 11,76 & 9,56 \\
\hline Classificação & & & \\
\hline
\end{tabular}

$\mathrm{Na}$ pesquisa realizada por Bezerra et al. (2014), estimando a ET diária para Mossoró, No Rio Grande do Norte, foram encontrados resultados para o método de Jensen-Haise classificados como muito bom, apresentado os coeficientes de Willmott (d), Pearson (e) e Confiabilidade (c) equivalentes a 88, 91 e 80\%, respectivamente, indicando que os resultados apresentados neste estudo apresentam uma boa representatividade para este método, assim como os resultados encontrados por Cavalcante Junior et al. (2011) em trabalho realizado na cidade de Mossoró avaliando métodos de estimativa da $\mathrm{ET}_{0}$ para as condições do semiárido Nordestino, caracterizado como ótimo, de acordo com a classificação proposta por Camargo e Sentelhas (1997).

Resultados similares foram encontrados por Fanaya Junior et al. (2012), em pesquisa realizada para avaliar os diferentes métodos empíricos para a estimativa da $\mathrm{ET}_{0}$ no município de Aquidauana no Estado de Mato Grosso do Sul, e por Cunha et al. (2013) tendo seus resultados classificados como mediano.

Para o método de $\mathrm{ET}_{0} \mathrm{HS}$, os resultados mostram que há subestimativa dos valores de $\mathrm{ET}_{0}$, tomando como referência o método de $\mathrm{ET}_{0} \mathrm{PM}$, em torno de 17,99\%, por isso, o valor calculado por este método foi corrigido

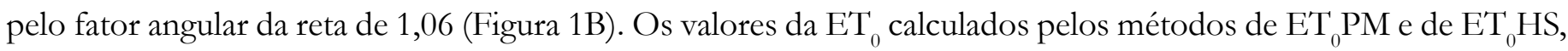
como também os valores simulados pelo ajuste da regressão linear (ET $\mathrm{PM}_{0}-\mathrm{ET}_{0} \mathrm{HS}$ ) podem ser observados na Figura 3. No ano de 2008, entre os meses de setembro à dezembro, a evapotranspiração médias foi de 7,56 \pm 0,69 calculado pelo método de ET $\mathrm{PM}, 6,2 \pm 0,40$ calculado pelo método de ET $\mathrm{H}_{0} \mathrm{HS}$ e de 7,56 \pm 0,43 calculado pelo método de $\mathrm{ET}_{0} \mathrm{HS}$ corrigida para ET $\mathrm{PM}$. Em 2009, obteve-se para este mesmo período, as seguintes médias 6,99 \pm 0,85, 6,18 \pm 0,43 e, 7,54 \pm 0,46 quando estimados pelos métodos de ET $\mathrm{PM}_{0} \mathrm{ET}_{0} \mathrm{HS}$ e ET $\mathrm{ES}_{0}$ corrigido para $\mathrm{ET}_{0} \mathrm{PM}$, respectivamente.

Os dados de evapotranspiração calculados pelo método de $\mathrm{ET}_{0} \mathrm{HS}$ e ajustados para o método de $\mathrm{ET}_{0} \mathrm{PM}$, para os anos de 2008 (Figura 3A) e de 2009 (Figura 3B), não foram aproximados satisfatoriamente (Tabela 1). O índice de concordância de d ficou abaixo de 0,71 , a correlação de $\varrho$ de 0,62 , o índice c abaixo de 0,44 e, elevada percentagem PD quando comparado com os demais métodos. 
Figura 3. Evapotranspiração de referência calculado pelos métodos de Penman-Monteith (-), PenmanHargreaves (----) e Hargreaves-Samani (…‥) nos anos de 2008 (A) e 2009 (B), Apodi-RN.
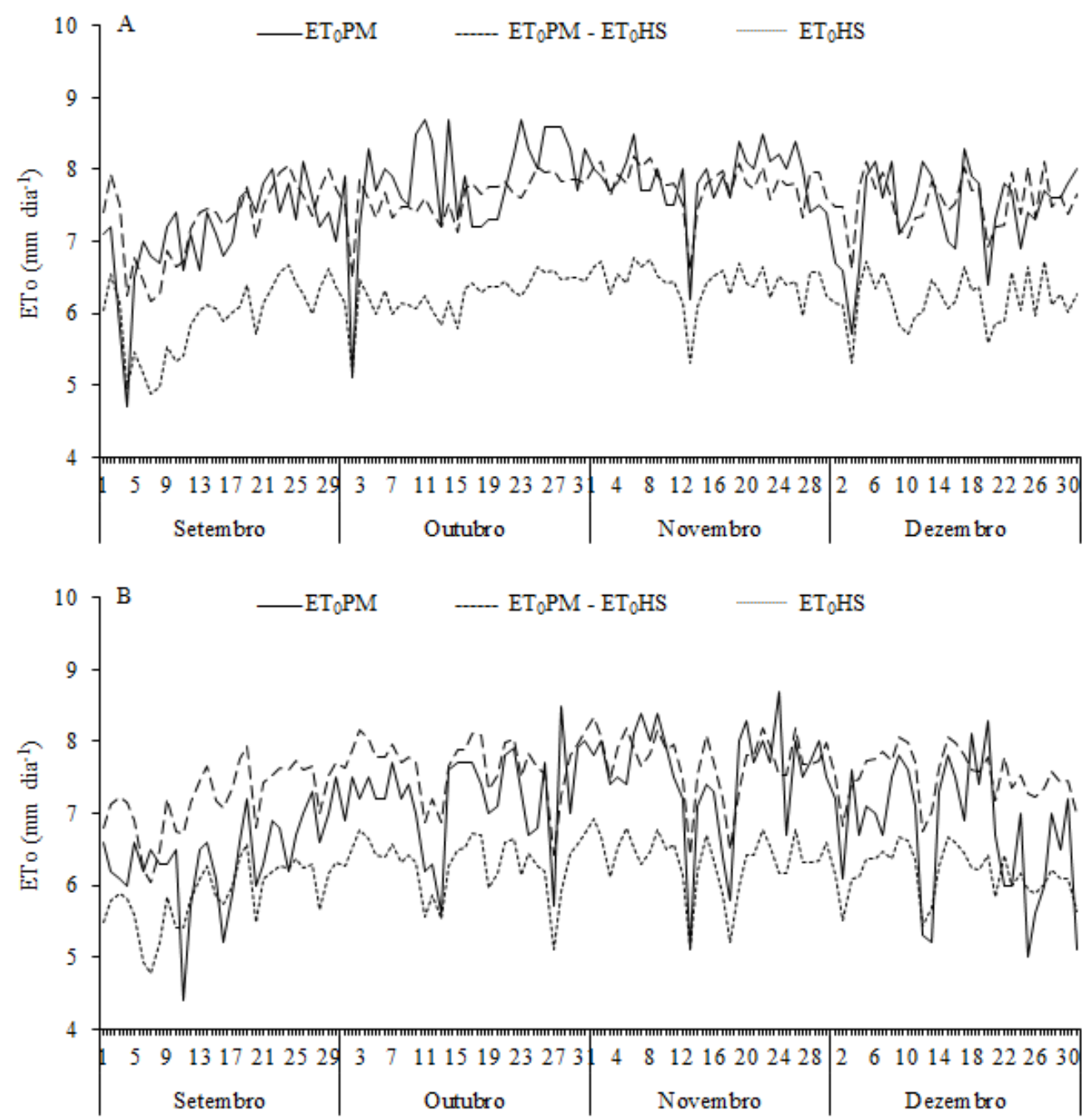

Chagas et al. (2013), realizando comparações entre métodos de estimativa da $\mathrm{ET}_{0}$ no município de Rio Real na Bahia, também encontraram resultados abaixo do padrão para os coeficientes d, @ e c com valores respectivos de 82, 72 e 59\% para o período seco, no entanto, os resultados para o período úmido foram de 93 , 88 e $82 \%$, concluindo que o método de ET $\mathrm{H}_{0} \mathrm{HS}$ foi o que obteve as avaliações mais baixas para todos os critérios, comparado com os demais métodos, tanto para o período seco como para o período úmido. Resultados semelhantes a este foram encontrados por Alencar et al. (2011) na região de Uberaba, em Minas Gerais, e por Cunha et al. (2013), classificados como mediano.

Diferentemente dos resultados apresentados, Fanaya Junior et al. (2012) encontraram valores respectivos iguais a 90, 85 e 77\%, classificado como muito bom, comprovando que não sofre influência significativa das demais variáveis utilizadas pelo método padrão para a região estudada.

$\mathrm{O}$ método de $\mathrm{ET}_{0} \mathrm{M}$ superestima os valores de $\mathrm{ET}_{0}$, tomando como referência o método de $\mathrm{ET}_{0} \mathrm{PM}$, em torno de 5,69\%, por isso, o valor calculado por este método foi corrigido pelo fator angular da reta de 0,68 (Figura 1C). $\mathrm{Na}$ Figura 3, podem ser observados os valores da $\mathrm{ET}_{0}$ calculados pelos métodos de $\mathrm{ET}_{0} \mathrm{PM}$ e de $\mathrm{ET}_{0} \mathrm{M}$, como também os valores simulados pelo ajuste da regressão linear (ET 0 PM - ET 0 M). No ano de 2008, entre os meses de setembro à dezembro, a evapotranspiração médias foi de 7,56 \pm 0,69 calculado pelo método de ET 0 PM, 7,99 $\pm 0,83$ calculado pelo método de $\mathrm{ET}_{0} \mathrm{M}$ e de 7,57 \pm 0,56 calculado pelo método de $\mathrm{ET}_{0} \mathrm{M}$ corrigida para $\mathrm{ET}_{0} \mathrm{PM}$. Em 2009, obteve-se para este mesmo período, as seguintes médias 6,99 \pm 0,85, 7,70 \pm 1,09 e, 7,37 \pm 0,74 quando estimados pelos métodos de ET $\mathrm{PM}_{0} \mathrm{ET}_{0} \mathrm{M}$ e ET $\mathrm{M}_{0}$ corrigido para $\mathrm{ET}_{0} \mathrm{PM}$, respectivamente. 
Os dados de evapotranspiração calculados pelo método de ET $\mathrm{E}_{0} \mathrm{M}$ e ajustados para o método de ET $\mathrm{PM}$, para os anos de 2008 (Figura 4A) e de 2009 (Figura 4B), não foram aproximados tão satisfatoriamente quando comparado com o método de $\mathrm{ET}_{0} \mathrm{JH}$ (Tabela 1). Porém foi melhor que a aproximação feita pelo método de ET $\mathrm{H}_{0} \mathrm{HS}$. O índice de concordância de d ficou acima de 0,84, a correlação de $\varrho$ acima de 0,76, o índice de desempenho c acima de 0,64 e, moderada percentagem PD.

Figura 4. Evapotranspiração de referência calculado pelos métodos de Penman-Monteith (-), PenmanMakkink (----) e Makkink (…...) nos anos de 2008 (A) e 2009 (B), Apodi-RN.
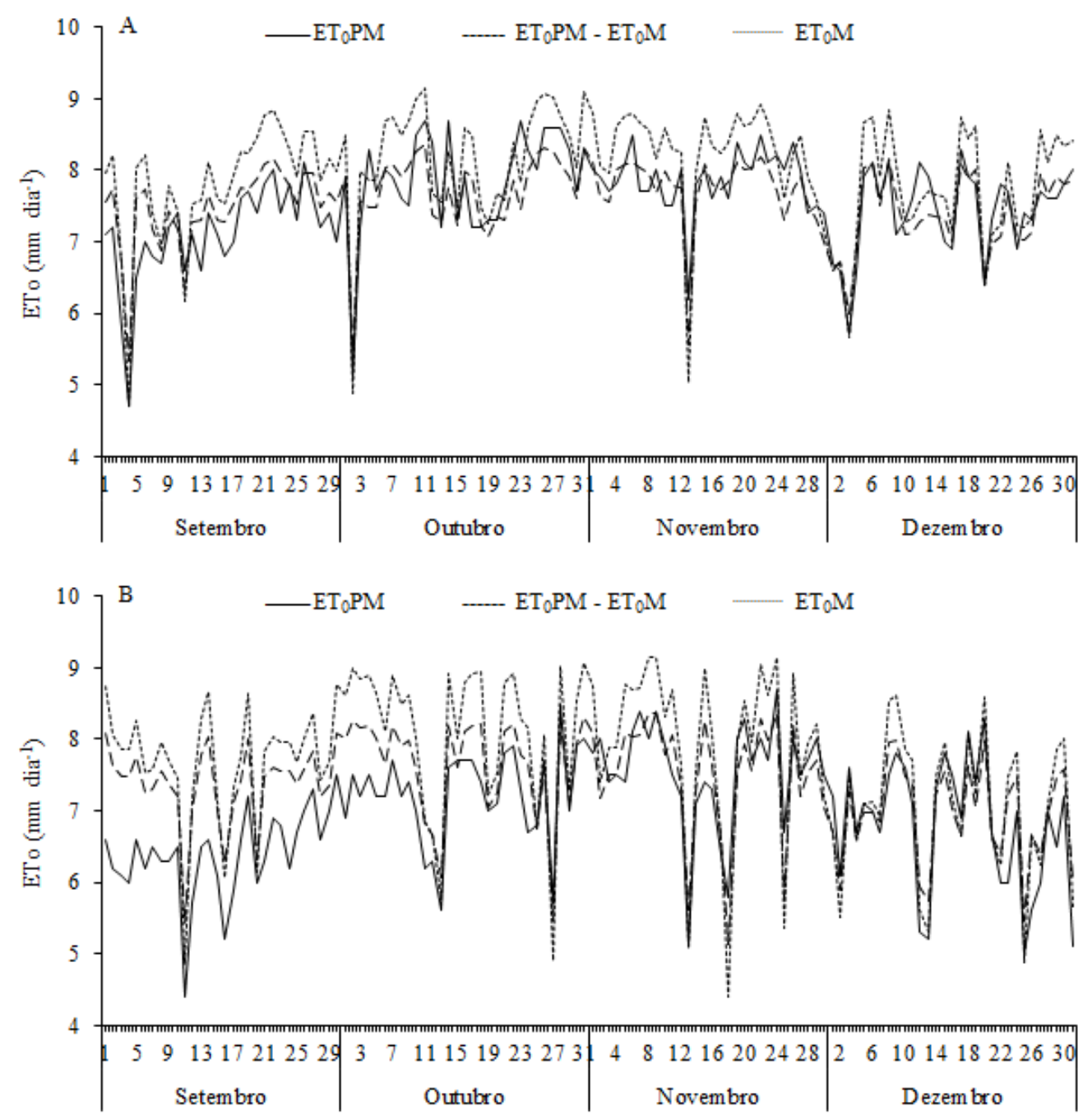

Bezerra et al. (2014), encontraram resultados favoráveis para este método, apresentado valores de d, @ e c iguais a 78, 87 e 68\%, classificado como bom, diferentemente dos resultados encontrados por Cavalcante Junior et al. (2011) e Cunha et al. (2013) que estão abaixo de 51\%, sendo classificados como péssimo e mau, respectivamente.

Entre os métodos utilizados para estimar a $\mathrm{ET}_{0}$, o método de $\mathrm{ET}_{0} \mathrm{M}$ é que diferencia menos dos dados obtidos por $\mathrm{ET}_{0} \mathrm{PM}$. Porém, quando se ajusta os dados calculados por ET $\mathrm{JH}$, obtêm-se uma boa aproximação do método padrão.

\section{Conclusão}

De acordo com os resultados encontrados, o método de Jensen-Haise torna-se o mais apropriado para estimar a $\mathrm{ET}_{0}$ na região de Apodi-RN entre os meses de setembro a dezembro, sendo o método que apresentou resultados mais aproximados do método de Penman-Monteith quando os valores são ajustados pelo fator 0,65 ; 
Pelo método de Makkink, obtém-se boa a aproximação dos valores estimados pelo método de PenmanMonteith, em Apodi-RN;

As estimativas da ET 0 obtido pelo método de Hargreaves-Samani são bastante distintas das obtidas pelo método de Penman-Monteith, tornando as variáveis de temperatura e umidade do ar insuficientes para a estimativa da $\mathrm{ET}_{0}$ na região de Apodi-RN, sendo indispensável variáveis como radiação solar e velocidade do vento que irão interferir no resultado final.

\section{REFERÊNCIAS}

Ayres M, Ayres-Júnior M, Ayres DL e Santos AAS. 2007. BioEstat: aplicações estatísticas nas áreas de ciências biométricas. Versão 5.0. Belém: Sociedade Civil Mamirauá, MCT-CNPq.

Alencar LP, Delgado RC, Almeida TS e Wanderley HS. 2011. Comparação de diferentes métodos de estimativa diária da evapotranspiração de referência para a região de Uberaba. Revista Brasileira de Ciências Agrárias, Recife, 6(2):337-343.

Allen RG, Pereira LS, Raes D and Smith M. 1998. Crop evapotranspiration -Guidelines for computing crop water requirements. Roma: FAO Irrigation and Drainage, (56):300.

Araújo WF, Costa SAA e Santos AE. 2007. Comparação entre métodos de estimativa da evapotranspiração de referência (ETo), Pará. Revista Caatinga, Mossoró, 20(4):84-88.

Bezerra JM, Moura GBA, Silva EFF, Lopes PMO e Silva BB. 2014. Estimativa da evapotranspiração de referência diária para Mossoró (RN, Brasil). Revista Caatinga, Mossoró, 27(3):211-220.

Camargo AP e Sentelhas PC. 1997. Avaliação do desempenho de diferentes métodos de estimativa da evapotranspiração potencial no estado de São Paulo. Revista Brasileira de Agrometeorologia, Santa Maria, 5(1):89-97.

Cavalcante Junior EGC, Oliveira AD, Almeida BM e Sobrinho JE. 2011. Métodos de estimativa da evapotranspiração de referência para as condições do semiárido Nordestino. Revista Ciências Agrárias, Londrina, 32:1699-1708.

Chagas RM, Faccioli GG, Aguiar Netto AO, Sousa IF, Vasco NA e Silva MG. 2013. Comparação entre métodos de estimativa da evapotranspiração de referência (ETo) no município de Rio Real-BA. Revista Irriga, Botucatu, 18(1):351-363.

Cunha FF, Magalhães FF e Castro MA. 2013. Métodos para estimativa da evapotranspiração de referência para Chapadão do Sul - MS. Revista Engenharia na Agricultura, Viçosa, 21(2):159-172.

Fanaya Junior ED, Lopes AS, Oliveira GQ e Jung LH. 2012. Métodos empíricos para estimativa da evapotranspiração de referência para Aquidauana, MS. Revista Irriga, Botucatu, 17(4):418-434.

Hargreaves GH and Samani, Z. Reference crop evapotranspiration from temperature. 1985. Journal of Applied Engineering in Agriculture, St Joseph, 1(2)96-99.

Jensen ME, Burman RD and Allen RG. 1990. Evapotranspiration and irrigation water requirements. New York: American Society of Civil Engineers, ASCE. Manual and Reports on Engineering Practices, 70:332.

Jensen ME, Haise HR. 1963. Estimating evapotranspiration from solar radiation. Journal of the Irrigation and Drainage

Division, ASCE, 89:15-41. 
Makkink GF. 1957. Testing the Penman formula by means of lysimeters. 1957. Journal of the Institution of Water Engineers, London, 11(3):277-288.

Penman HL. 1948. Natural evaporation from open water, bare soil and grass. Proceedings of the Royal Society of London. Series A. Mathematical and Physical Sciences, London, 193:120-145.

Pereira AR, Villa Nova NA e Sediyama GC. 1997. Evapo(transpi)ração. Piracicaba: FEALQ, 1997. 1:183

Thornthwaite GW. 1948. Na approach toward a rational classification of climate. Geographycal Review, 38(1):55-94.

Willmott CJ. 1981. On the validation of models. Physical Geography, 2:184-194. 